\title{
WebPensa: Uma Ferramenta Para Apoio ao Desenvolvimento do Pensamento Crítico
}

\author{
Scheila Wesley Martins ** \\ Augusto César Melo de Oliveira * \\ Leandro José Komosinski
}

\begin{abstract}
Resumo
Este trabalho apresenta um ambiente educacional construído para auxiliar a desenvolver o pensamento crítico, cujo uso estimule alunos e professores ao envolvê-los em uma nova dinâmica na qual a o diálogo, a reflexão e a troca intelectual se desenvolva como processo de aquisição do conhecimento. O WebPensa é uma ferramenta mediadora da Tecnologia Computacional Educativa, e apenas um dos resultados possíveis, baseados em um modelo computacional desenvolvimento e inspirado na metodologia de aprendizado definida por Matthew Lipman e nas premissas da Teoria da Atividade.

Palavras-Chave: Tecnologia Educacional, Pensamento Crítico, Teoria da Atividade.

Abstract

This work presents an educational environment implemented to assist to develop the critical thinking, whose use stimulates students and teachers to involve it them in a new dynamics in which to the dialogue, the reflection and the intellectual exchange be develops as process of acquisition of the knowledge. The WebPensa is a mediating tool of the Educative Computational Technology, and only one of the possible results based in a computational development and inspired model in the methodology of learning defined for Matthew Lipman and in the premises of the Theory of the Activity.
\end{abstract}

Keywords: Educational Technology, Critical Thinking, Activity Theory.

\section{Introdução}

Os resultados apresentados pelo Programa Internacional de Avaliação de Estudantes (PISA) realizado pela UNESCO com objetivo de verificar a qualidade do ensino mundial nas áreas de leitura, matemática e ciências alertou para urgente busca por melhorias na preparação intelectual e cultural dos estudantes brasileiros (INEP,2002)(INEP,2003). O baixo desempenho da maioria dos estudantes nas duas edições do exame reacendeu os debates quanto aos problemas do aprendizado fragmentado (IVANISSEVIC,2003) e da necessidade de atualização do modelo educacional e dos profissionais da educação do Brasil (ARANHA,1996) (FREIRE,1996).

Professores, de todas as áreas do conhecimento e de todos os níveis escolares, constatam que a falência do ensino fundamental e médio se reflete em maiores dificuldades para o cumprimento dos programas curriculares. Observa-se com mais perplexidade o declínio qualitativo das capacidades críticas, criativas e motivacionais dos ingressos às universidades. Impressionam a falta de iniciativa e incapacidade dos

\footnotetext{
"Software desenvolvido a partir da dissertação "Um Modelo Computacional Para Apoiar o Pensamento Crítico", PPGCC/UFSC, defendido em 23/05/2005.

" Mestre em Computação (PPGCC/UFSC), Especialista em Informática em Educação (UFLA), Bacharel em Ciência da ... Computação (UFMT) Professora Colaboradora (UDESC) e (FCJ), scheila@inf.ufsc.br, http://www.inf.ufsc.br/ scheila. Bacharel em Ciência da Computação (UFAL), Mestrando (PPGCC/UFSC), acmo@inf.ufsc.br, http://www.inf.ufsc.br/ acmo. Doutor em Engenharia de Produção (UFSC), Professor Adjunto (INE/UFSC), leandro@inf.ufsc.br, http://www.inf.ufsc.br/ leandro V. $3 \mathrm{~N}^{\mathrm{o}} 1$, Maio, 2005
} 
alunos de interpretar, de abstrair e de acompanhar a lógica e a dinâmica da maioria das disciplinas (ZANNELLA,2003). Muito embora, o pragmatismo sobre a eficiência e a eficácia de ferramentas da tecnologia educacional seja uma constante entre os institutos de educação e de tecnologia (SETZER,2001) (PAIS,2002).

A Tecnologia Educacional possui requisitos que permitem a produção de ferramentas que possibilitam o desenvolvimento do raciocínio de maneira reflexiva. Basta que acreditemos no potencial da tecnologia como artefato de mediação, ao se explorar diretivas para atividades de aprendizagem reflexiva, e desde que a tecnologia seja aplicada como suporte a um contexto educacional renovado (NIKERSON,1995) (BELLAMY,1996). Atualmente, as novas propostas tencionam desistigmatizar os objetivos e as expectativas do uso das ferramentas da tecnologia educacional, deixandoas cumprir o papel que lhes cabe no processo ensino-aprendizagem: artefato de mediação.

\section{Tecnologia e Educação}

O receio e o descrédito que vários estudiosos dão às ferramentas computacionais educativas repousa, quase sempre, sob um excesso de expectativas e nas frustrações dos resultados em torno do uso do computador nas salas de aula (REYNOLDS et. al.2003). $\mathrm{O}$ fato é que se ignora que toda tecnologia educacional constitui um meio, e não um fim em si mesma. Portanto, não podemos responsabilizá-la unicamente pela melhora ou não do processo de aprendizagem (VALENTE, 1993).

Tradicionalmente, os softwares educativos implementam as práticas correntes nas salas de aulas, e estas se baseiam em alguma teoria de aprendizagem (RAMOS,1999). Então, é importante observar os contrastes nas influências exercidas pela teoria comportamentalista e as diversas teorias interacionistas, que até hoje são as práticas pedagógicas mais comuns, e as grandes influências enquanto domínio de modelagem dos softwares educativos (VALENTE,1993) (SANTOS,1999):

- $\quad$ Os programas Tutoriais, Exercício e Prática (drill-and-practice), os jogos e softwares de simulações que abusam de atividades mentais de repetição e memorização para que o usuário avance em suas etapas, são abordagens influenciadas pela teoria de Skinner;

- Os ambientes de hipermídia inteligentes que aliam as construções de hipertextos e hipermídia com as prerrogativas da Inteligência Artificial, as chamadas hipermídia de navegação ou apresentação adaptativa, freqüentemente implementam as premissas da "Hierarquia de Aprendizagem" proposta por Gagné;

A linguagem LOGO e as ferramentas de aprendizagem baseadas em resolução de problemas são influências do construtivismo de Piaget;

- As ferramentas de aprendizagem significativa, como os mapas de conceitos, se referem à influência exercida pela teoria de Ausubel e Novak, enquanto as ferramentas que se baseiam na aprendizagem por descobertas em ambientes de hipermídia educacionais, em grandes bases de dados e na Internet tem influência do cognitivismo de Bruner;

Os ambientes distribuídos que se valem da aprendizagem cooperativa se baseiam nas premissas sócio-interacionistas de Vygotsky;

Embora o desenvolvimento de software educacional esteja baseado ou no comportamentalismo ou nas ciências cognitivas, ainda são poucos os desenvolvedores que se utilizam de outros fundamentos teóricos, especialmente a Teoria da Atividade, pois esta é ainda pouco conhecida. 


\section{Novas Premissas Teóricas Como Domínio de Aplicação Educacional}

A Pedagogia do Julgamento (LIPMAN,1995) se propõe a "ensinar a pensar" utilizando o discurso filosófico (ensino socrático). Seu objetivo é um desenvolvimento contínuo do pensamento ao longo do processo educacional formal, partindo do estímulo à capacidade de bem julgar. Emitir um julgamento é um comportamento que está na base para o desenvolvimento qualitativo das habilidades cognitivas de mais alto nível, constituindo uma unidade elementar do pensamento. $\mathrm{O}$ julgamento constitui a unidade cognitiva básica para o desenvolvimento tanto do pensar crítico quanto do pensar criativo, de maneira que é a base destes. O Julgamento sofre a influência tanto dos critérios quanto dos valores individuais, que constituem respectivamente, o elemento racional e o elemento afetivo de sua composição.

A Teoria da Atividade (ENGESTROM et. al. 1999) determina uma nova concepção do processo ensino-aprendizagem, no qual diversos elementos, individuais e coletivos, colaboram para que o aluno conscientemente o entenda como uma experiência totalmente significativa. A prática pedagógica inspirada nessas premissas proporciona ao ambiente educacional uma dinâmica diferenciada (BELLAMY,1996), na qual são igualmente importantes tanto o processo de aquisição quanto $o$ conhecimento em si, mesclando a participação ativa dos componentes da comunidade escolar com os espaços necessários para o monitoramento, a reflexão, a construção, a correção e a auto-correção do conhecimento em construção.

A escolha da atividade de aprendizagem Crítica do Conhecimento (KOMOSINSKI,2000) se deve ao fato de que ela está em grande sintonia com os objetivos almejados pela Pedagogia do Julgamento. A crítica do conhecimento proporciona momentos no qual o aluno pode exercitar o desenvolvimento do pensamento através da reflexão (individual ou coletiva), durante a resolução de problemas. Outras habilidades cognitivas como a dedução, a inferência, o questionamento e a autocorreção do conhecimento, impírico e ampliado, são também favorecidas. A crítica do conhecimento permite ao aluno reconstruir e aprimorar não apenas suas significações sobre o contexto do problema em foco, como também estimula e oportuniza o exercício dos recursos da linguagem para o desenvolvimento de sua argumentação.

\section{Um Modelo Computacional Para o Pensamento Crítico}

Várias propostas que objetivam mensurar e desenvolver o pensamento crítico a partir de ambientes computacionais foram implementadas (NEWMAN,1995)(DAUD e HUSIN,2004). Todo domínio de aplicação educacional acaba por exigir dos desenvolvedores uma nova perspectiva do processo de modelagem e produção de software, nesse caso, particularmente em função do comprometimento com uso de abordagens teóricas.ainda não muito exploradas.

A maior relevância dessa proposto está nas inspirações pedagógicas ligadas ao desenvolvimento do pensamento crítico, concentrando-se em especificar entre os elementos da Pedagogia do Julgamento, aqueles que fariam parte do modelo, o que determinou uma redução necessária à definição de um modelo computacionalmente viável.

O modelo desenvolvido, ilustrado na figura 1, se baseia em uma simplificação da teoria de Lipman, cuja a relação entre os componentes do modelo é orientada segundo as premissas da Teoria da Atividade e no exercício da atividade de Crítica do Conhecimento através da resolução de problemas propostos a pequenos grupos de estudo. 


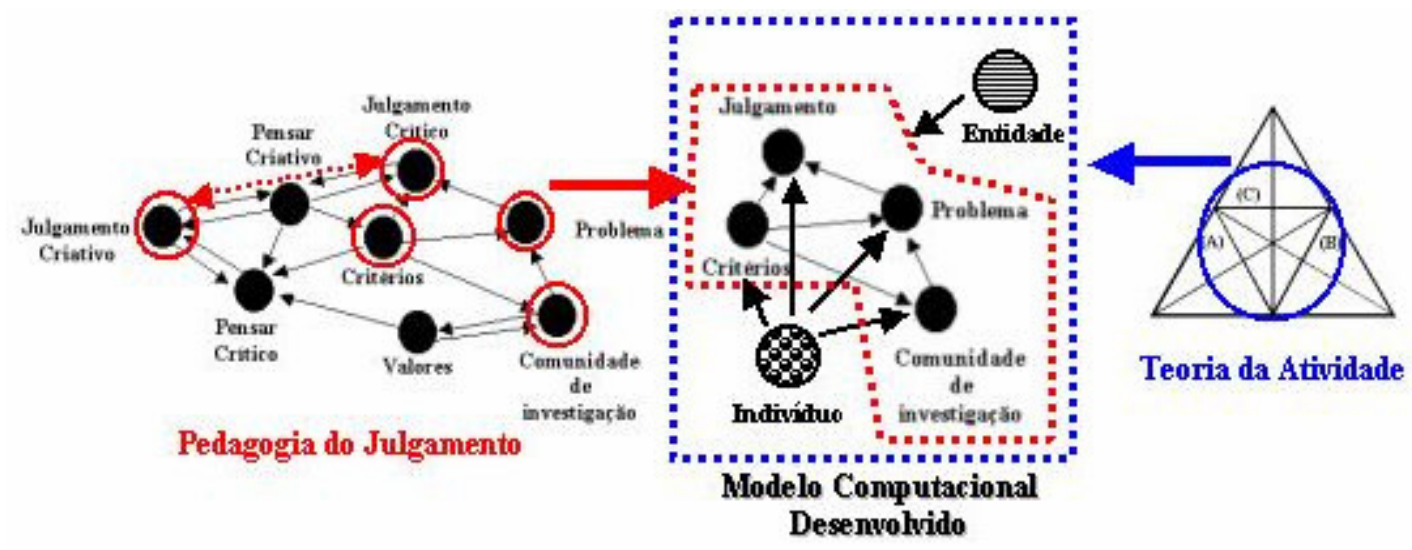

Figura 1. Abstração do domínio para desenvolver o modelo proposto

\section{O Ambiente WebPensa}

O WebPensa é uma implementação a partir do modelo desenvolvido e busca disponibilizar um ambiente através da Web que permita apoiar o desenvolvimento do pensamento crítico através de discussões sobre a resolução de problemas, do conteúdo de qualquer disciplina, do ensino fundamental ao universitário.

A priori, o WebPensa poderia ser adotado em qualquer contexto no qual a questão "justifique sua resposta" pudesse ser efetuada. Desde que essa resposta, não necessariamente, esteja associada à demonstrações de provas matemáticas formais, uma vez que estas constituem fatos dos quais a discordância implica, obviamente, em erro.

Questionamentos onde há a necessidade de emissões dessa natureza podem não ser resultado de reflexões, mas de um processo de memorização de demonstrações obtidas das bibliografias relativas ao conteúdo ou problema especificado.

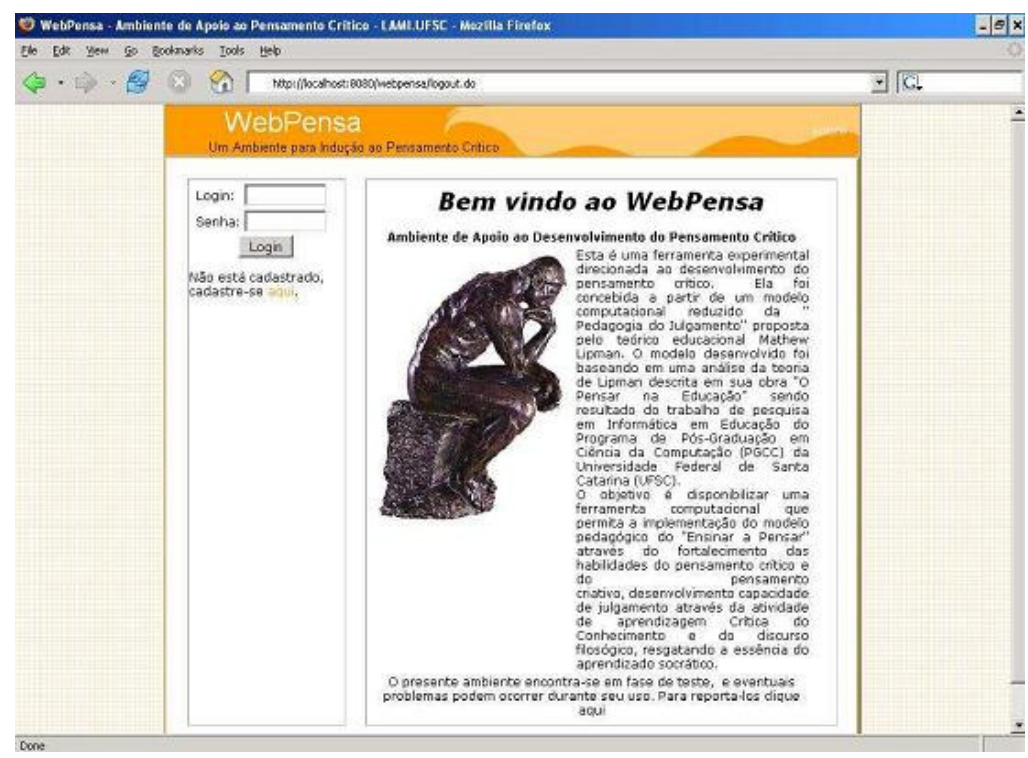

Figura 2. Tela Principal do WebPensa 


\section{Características da Arquitetura e Implementação do WebPensa}

Dentro das características técnicas, o protótico foi desenvolvido na linguagem de programação JAVA e outras linguagens (XML, JSP, CSS, HTML) utilizando o Apache Struts (APACHE, 2004), um framework para aplicações Web baseado na abordagem Model2 que é uma variação padrão Model View Controller (MVC).

Dessa forma a implementação do ambiente é dividido em camadas. No qual, a Camada Modelo apresenta a modelagem das entidades do pensamento crítico. As camadas de Controle e Visão são as que interagem diretamente com o usuário do sistema, nas quais cada ação é realizada pela Camada de Controle que retorna com o resultado das ações para a camada Visão que apresenta as informações para o usuário. A Camada de Persistência usa o padrão DAO, guardando os estados dos objetos da camada Modelo no banco de dados, o qual no caso, foi escolhido o MySql. A figura 3 mostra a arquitetura utilizada na implementação do WebPensa.

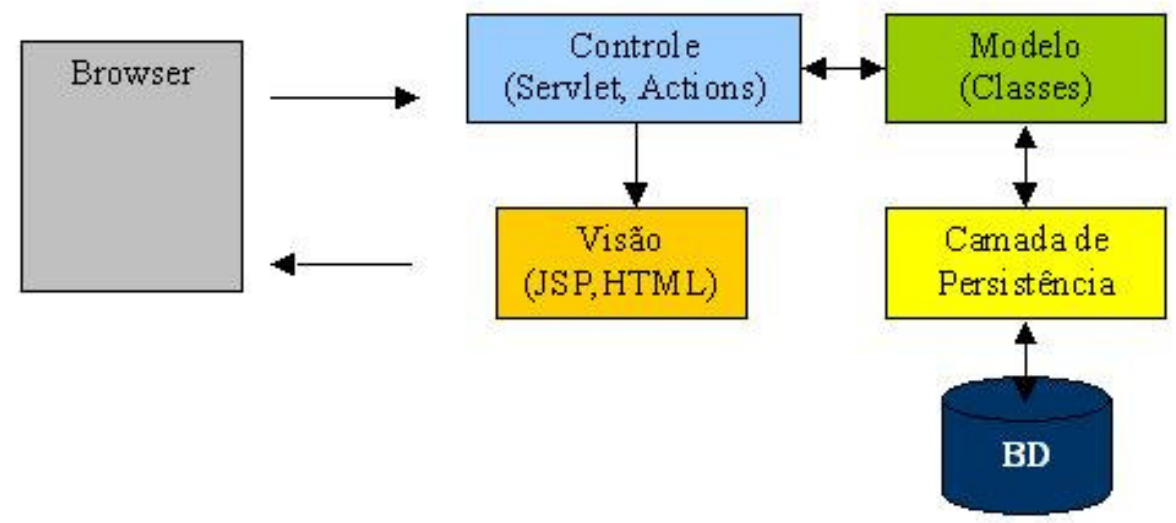

Figura 3. Arquitetura em camadas no WebPensa seguindo o Model2.

\section{Características Pedagógicas do WebPensa}

A atividade de crítica ao conhecimento é realizada no contexto das Comunidades de Investigação onde a dinâmica da aprendizagem se desenvolve como um processo investigativo, através de procedimentos que tem por objetivo estimular e exercitar tanto a racionalidade quanto a criatividade dos participantes. A figura 4 mostra uma Comunidade de Investigação no WebPensa.

Assim, a Comunidade de Investigação constitui-se de membros envolvidos em atividades educacionais a serem desenvolvidas, de maneira colaborativa, a partir de ações como: o intercâmbio de idéias, questionamentos, inferências, deduções e autocorreções. Todas essas ações são desenvolvidas a partir da interação dos componentes da comunidade através do diálogo filosófico e da reflexão individual e/ou coletiva. Cada Comunidade de Investigação tem um problema específico que constitui a temática da atividade de Crítica do Conhecimento proposta aos membros da comunidade, para que estes voltem sua atenção e seus esforços para sua resolução a partir do diálogo e da reflexão (individual/coletiva), definindo o elemento que inicia a dinâmica da Pedagogia do Julgamento proposta por Lipman.

A discussão sobre o problema faz com que os membros da comunidade de investigação identifiquem critérios, que sirvam de base do mais alto grau de confiabilidade, para nortear considerações decisivas e orientadoras de qualquer tentativa de classificação ou avaliação de suas argumentações, com as quais os membros da comunidade de investigação se identificariam e reconheceriam como fatores V.3 $\mathrm{N}^{\circ} 1$, Maio, 2005 
predominantes no julgamento crítico. Assim, cada membro da comunidade pode emitir seu julgamento (crítica ao conhecimento) sobre o problema, baseando-se em determinados critérios que o membro escolhe como relevantes para o seu julgamento, dentre os critérios presentes na comunidade de investigação.

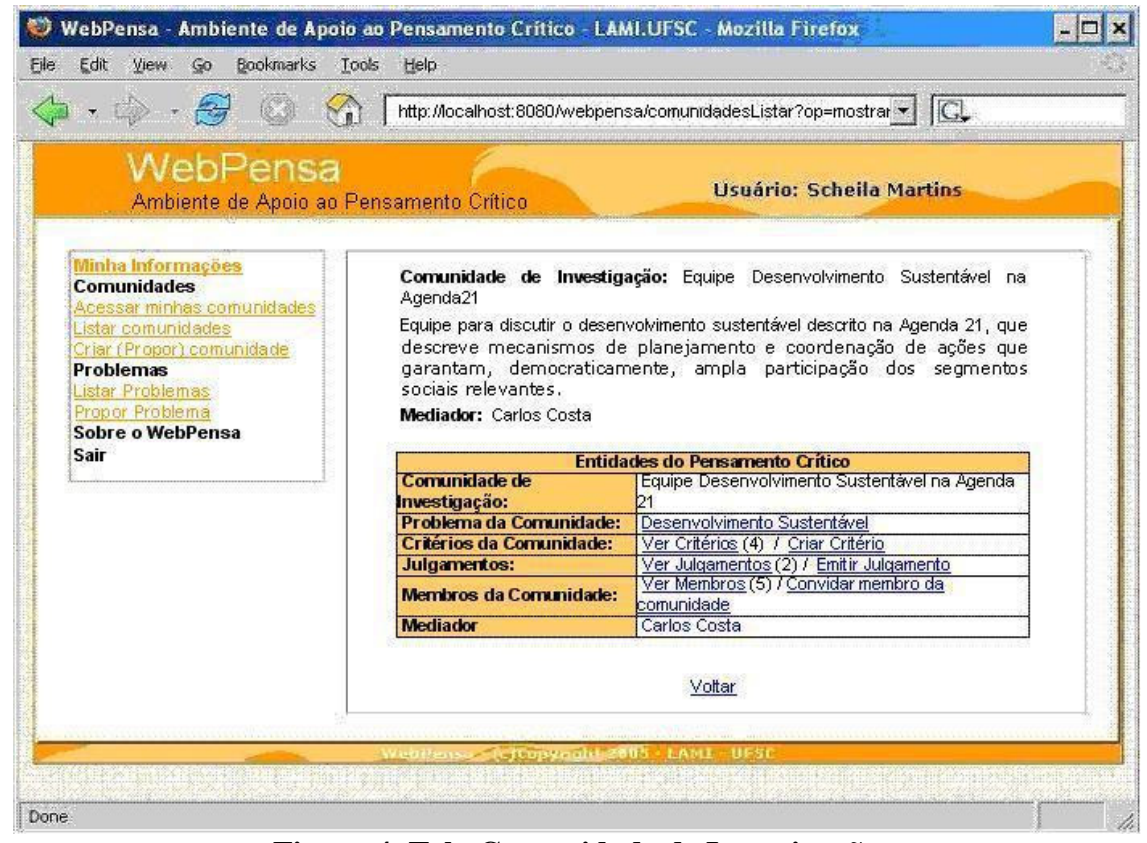

Figura 4. Tela Comunidade de Investigação

O julgamento possui uma relação com o exercício do questionamento de tal forma, que podemos entender essa relação questionamento/julgamento análoga à relação processo/produto, muito embora esse seja um tipo de ligação não exclusiva. Da lista de julgamentos emitidos pela Comunidade de Investigação, cada membro pode, também, avaliar e opinar sobre o julgamento do outro. Os membros podem se associar aos julgamentos de outros membros, concordando ou discordando da versão do julgamento emitido, considerando os critérios usados nesse determinado julgamento. Assim, o membro que emitiu o julgamento pode emitir novas versões do seu julgamento. Dessa forma é possível acompanhar o histórico de evolução da interação entre os membros, do ponto de vista de cada julgamento emitido por cada um dentro da Comunidade de Investigação.

\section{Conclusão}

O ambiente WebPensa, constitui um exemplo de pesquisa em Tecnologia Computacional Educativa com propósito de estimular uma mudança na interação entre alunos e professores, dinamizando o processo educacional pelo estímulo ao debate de idéias, à construção de argumentações, ao exercício do discurso e à valorização do processo de aquisição do conhecimento.

É fato que o uso de ferramentas computacionais não resolverão os inúmeros problemas educacionais, sem que antes, tanto a educação quanto os educadores também passem por transformações. Reformas que valorizem e priorizem a qualidade do ensino e da formação dos profissionais do ensino, modificando a visão ultrapassada do analfabetismo funcional, uma vez que se permite a inúmeros jovens passarem e 
avançarem pelos diversos níveis do processo formal de ensino, sem desenvolver minimamente as próprias habilidades de pensamento.

A tecnologia trás para as salas de aulas grandes possibilidades de dinamização e, pode mesmo, facilitar a apropriação de uma nova metodologia de aprendizagem, mas não pode ser responsabilizada unicamente por atingir esses objetivos, sem que haja o esforço efetivo de professores e alunos. Para um bom resultado do uso do computador em sala, o professor deve estar consciente do seu compromisso como educador. Aos estudantes também cabem responsabilidades, mas é preciso orientá-los para que eles se desacostumem do aprendizado passivo. No entanto, o processo escolar desarticulado e enfadonho é uma realidade, uma prática cíclica que há anos se mantém inerte. Rompê-la depende de atitudes que foram desestimuladas nos estudantes desde muito cedo.

Assim como outras ferramentas educacionais existentes, não se pode assegurar que se consiga atingir os objetivos propostos por Lipman, sem que haja contribuições de estudantes e professores. Até porque o WebPensa, é uma ferramenta de mediação, idealizado propositalmente como um elemento de motivação. De fato, só poderemos garantir que o WebPensa tenha atingido os objetivos a que se propôs quando, ele for desnecessário. Isto é, quando a metodologia de aprendizagem proposta por Lipman já tenha sido totalmente assimilada e incorporada ao cotidiano da sala de aula, ou seja, o estudante conscientemente troque a memorização pela reflexão sobre o conteúdo.

Importante esclarecer que isso não implica que o WebPensa tenha determinado um esforço de modelagem e implementação computacional inútil, pois a análise dos fundamentos teóricos do domínio não foi uma atividade trivial. Um dos desafios encontrados foi estabelecer um equilíbrio entre as premissas pedagógicas do modelo e as restrições de implementação. Mais de uma vez, foi preciso repensar as estratégias de implementação para que se preservasse o máximo dos aspectos conceituais do modelo, sem incorrer em um aumento excessivo de complexidade computacional. Isso implica dizer que, o WebPensa poderia ser completamente diferente, em face das decisões de implementação tomadas.

Atualmente, esse protótipo do WebPensa já se encontra em nível funcional e disponível para teste. A próxima etapa é fazer sua aplicação em um cenário educacional real, no qual se possa avaliar a validade do modelo computacional desenvolvido para a metodologia de Matthew Lipman, bem como identificar se os objetivos propostos foram atingidos, e ainda, em que níveis qualitativos.

\section{Referências}

APACHE. The Apache Software Foundation. The Apache Struts Web Application Framework. Disponível em <http://struts.apache.org/> acessado em 02/05/2004.

ARANHA, M. L. de A. Filosofia da Educação. $2^{a}$ edição, pgs. 148-181. Ed. Moderna: São Paulo, 1996.

BELLAMY, R. K. E. Designing Education Technology: Computer-Mediated

Change. In: NARDI, B. (Edited by). A. Context and Consciosness: Activity Theory and Human-Computer Interaction, pgs. 123-146. The MIT Press: Massachusetts, 1996.

DAUD, N. M. and HUSIN, Z. Developing Critical Thinking Skilss In Computer-

Aided Extended Reading Classes. Bristish Journal of Education Technology, Vol. 35, No. 04, pgs.477-487, 2004.

ENGESTRÖM, Y.; MIETTINEN, R. and PUNAMÄKI, R. Perspectives on Activity

Theory. In: Learning in Doing: Social, Cognitive and Computational Perspectives, (edited by), Cambridge University Press, 1999. 
FREIRE, P. Pedagogia da Autonomia: Saberes Necessários à Prática Educativa. Coleção Leitura. Ed. Paz e Terra: São Paulo, 1996.

INEP. Instituto Nacional de Estudos e Pesquisas Educacionais. Relatório Nacional PISA 2000. Ministério da Educação do Brasil (MEC): Brasília/DF, 2002. Disponível em <http://www.inep.gov.br/download/internacional/pisa/pisa2000.doc $>$ acessado em 10/10/03 às 19:27.

INEP. Instituto Nacional de Estudos e Pesquisas Educacionais. Relatório Completo PISA 2003. Ministério da Educação do Brasil (MEC): Brasília/DF, 2003. Disponível em <http://www.pisa.oecd.org/Docs/download/pisaplus_eng01.pdf $>$ acessado em 10/10/03 às 19:27.

IVANISSEVICH, A. Saber Fragmentado: Um Retrato do Conhecimento Científico de Nossos Jovens. Revista Ciência Hoje, Vol. 34, No. 200, pgs. 26-33, Dezembro, 2003.

KOMOSINSKI, L. J. Um Novo Significado para a Educação Tecnológica Fundamentado na Informática como Artefato Mediador da Aprendizagem. (Tese de Doutorado). Programa de Pós-Graduação em Engenharia de Produção da UFSC, Dezembro, 2000.

LIPMAN, M. O Pensar na Educação. Ed. Vozes: São Paulo, 1995.

NEWNAM, R. D. and WEBB, B. and COCHRANE, C. A Content Analysis Method to Measure Critical Thinking in Face-to-Face and Computer Supported Group Learning. In: IPCT-J, Vol. 3, No. 2, pgs 56-77, April, 1995. Disponível em $<$ http://www.qub.ac.uk/mgt/papers/methods/contpap.html> acessado em 05/04/04 às 10:00.

NICKERSON, R. S. Can Technology Help Teach for Understanding?, In: Software Goes to School: Teaching for Understanding with New Technologies, Chaper 1, section "Understanding Understanding". (Edited by) David N. Perkins et al., Oxford University Press: New York, 1995.

PAIS, L. C. Educação Escolar e as Tecnologias da Informática. Ed. Autêntica: Belo Horizonte, 2002.

RAMOS, E. M. F. Análise Ergonômica do Sistema HiperNet Buscando o Aprendizado da Cooperação e da Autonomia. (Tese de Doutorado). Programa de Pós Graduação em Engenharia Produção e Sistemas da UFSC. Novembro, 1996.

REYNOLDS, D.; TREHARNE, D. and TRIPP, H. ICT: The Hopes and the Reality. In: Bristish Journal of Education Technology, Vol. 34, No. 2, pgs. 151-167, 2003.

SANTOS, N. Desenvolvimento de Software Educacional. DCC/IME/UERJ: Rio de Janeiro, 1999. Disponível em <http://www.ime.uerj.br/neide/DesSoft.html > acessado em 04/10/2004.

SETZER, V. W. Meios Eletrônicos e Educação: Uma Visão Alternativa. Coleção Ensaios Transversais, Ed. Escrituras: São Paulo, 2001.

VALENTE, J. A. (Org.). Computadores e Conhecimento: Repensando a Educação. Ed.(NIED/UNICAMP): Campinas/SP, 1993.

ZANELLA, J. L. Dilemas de um Professor em Sala de Aula na Busca de um Ensino Reflexivo. Evento Educador2000 - Congresso Internacional de Educação. São Paulo, 2000. Disponível em <http://www.edutec.net/Textos/Alia/MISC/jlzanella.htm> acessado em 02/05/2003. 